\title{
Results on some special functions related to primes and a lemma regarding Carmichael's conjecture
}

Abhishek Das ( $\square$ abhik.das31@gmail.com )

B M Birla Science Centre, Hyderabad, India https://orcid.org/0000-0002-3815-3382

\section{Research Article}

Keywords: Primes, Riemann Zeta function, Von Mangoldt function, Chebyshev functions, Prime counting function

Posted Date: February 28th, 2022

DOI: https://doi.org/10.21203/rs.3.rs-1404183/v1

License: (9) This work is licensed under a Creative Commons Attribution 4.0 International License. Read Full License 


\title{
Results on some special functions related to primes and a lemma regarding Carmichael's conjecture
}

\author{
Abhishek Das \\ B. M. Birla Science Centre \\ Adarsh Nagar, Hyderabad \\ India, 500063 \\ abhik.das31@gmail.com
}

\begin{abstract}
In this paper, we prove a few lemmas and theorems pertinent to special functions like the Riemann zeta function, the Von Mangoldt function, the Euler totient function and the prime counting function, all of them being related to primes.
\end{abstract}

\section{Introduction}

The present paper indulges in some special functions that are related to primes and thereby we prove a few significant and novel results. The central theme of the paper deals with prime numbers, their properties, the prime counting function and so on.

The current article is divided into five sections; the second section focuses on lemmas and theorems pertinent to the Riemann zeta function and the Von Mangoldt function, the third section encompasses the first Chebyshev function and Euler's totient function, the fourth section concerns the prime counting function and in the final section the results of the paper are summarized.

\section{The zeta function and the Von Mangoldt function}

The Riemann zeta function $[1,2]$ has widespread significance in various fields of mathematics. We prove a few interesting identities and results in this section that are related to this special function and also the Von Mangoldt function [3].

Lemma 2.1. Given the derivative of the zeta function

$$
\zeta^{\prime}(s)=-\sum_{n=2}^{\infty} \frac{\ln n}{n^{s}}
$$

it can also be expressed as

$$
\zeta^{\prime}(s)=-\zeta(s) \sum_{p} \frac{\ln p}{p^{s}}-\delta
$$

where, $\delta=\ln f(p)$, $p$ denoting primes and $f(p)$ being a function of $p$.

Proof. From equation (1) we have

$$
\zeta^{\prime}(s)=-\sum_{n=2}^{\infty} \ln (n)^{\frac{1}{n^{s}}}=-\ln \left[\prod_{n=2}^{\infty}(n)^{\frac{1}{n^{s}}}\right]
$$


Now, let's take a look at the infinite product

$$
\prod_{n=2}^{\infty}(n)^{\frac{1}{n^{s}}}=(2)^{\frac{1}{2^{s}}} \cdot(3)^{\frac{1}{3^{s}}} \cdot(4)^{\frac{1}{4^{s}}} \cdots
$$

which can be rewritten as

$$
(2)^{\frac{1}{2^{s}}} \cdot(3)^{\frac{1}{3^{s}}} \cdot(2 \cdot 2)^{\frac{1}{4^{s}}} \cdot(5)^{\frac{1}{5^{s}}} \cdot(2.3)^{\frac{1}{6^{s}}} \cdots(3.3)^{\frac{1}{9^{s}}} \cdot(2.5)^{\frac{1}{1^{s}}} \cdots
$$

Continuing the process infinitely we will obtain the following fragmented product series

$$
\left\{(2)^{\frac{1}{2^{s}}}\right\}^{1+1 / 2^{s}+1 / 3^{s}+\cdots} \cdot\left\{(3)^{\frac{1}{3^{s}}}\right\}^{1+1 / 2^{s}+1 / 3^{s}+\cdots} \cdot\left\{(5)^{\frac{1}{5^{s}}}\right\}^{1+1 / 2^{s}+1 / 3^{s}+\cdots} \cdots \cdot f(p)
$$

where, $p$ denotes the primes numbers and $f(p)=2^{\frac{1}{4^{s}}} \cdot 3^{\frac{1}{9^{s}}} \cdot 2^{\frac{1}{10^{s}}} \ldots$ (which is an infinite product, ostensibly). Thus, the product that we started with can be expressed as

$$
\prod_{n=2}^{\infty}(n)^{\frac{1}{n^{s}}}=\prod_{p}\left[\left\{p^{1 / p^{s}}\right\}^{\zeta(s)} \cdot f(p)\right]
$$

Thus we have

$$
\zeta^{\prime}(s)=-\ln \left[\prod_{p}\left[\left\{p^{1 / p^{s}}\right\}^{\zeta(s)} \cdot f(p)\right]\right.
$$

It is to be noted that, though $\zeta(s)=\prod_{p} \frac{1}{1-p^{-s}}$, it is independent of the multiplicand terms $p^{1 / p^{s}}$ in the product, of which it is the exponent. Thus, we finally have

$$
\zeta^{\prime}(s)=-\zeta(s) \sum_{p} \frac{\ln p}{p^{s}}-\delta
$$

where, $\delta=\ln f(p)$ is the remainder term.

Remark. It is easy to see that we will have

$$
f(p)=\prod_{p} p^{l^{s} / r^{s}}
$$

where, $l^{s}=\sum_{I \in Z^{+}} j_{i}^{s} I^{s}$ is the infinite sum of the $s-$ th exponents of maybe not all, but an infinite number of positive integers with some undetermined multiplicity $j_{i}^{s}$ and $r^{s}=\prod_{p}\left(p^{k_{i}}\right)^{s}$ is the infinite product of the $s$ - th exponents of all primes with some undetermined multiplicity $k_{i}$.

Theorem 1. The infinite product of all prime numbers or the infinite primorial is equal to the infinite product of all natural numbers or the infinite factorial and they attain the value of $\sqrt{2 \pi}$ :

$$
p \#=N !=\sqrt{2 \pi}
$$

where, $p \#=\prod_{p} p$ and $N !=\prod_{n} n$ represent the infinite primorial and the infinite factorial respectively.

Proof. We know that the formula $N !=\sqrt{2 \pi}$ originates from Lerch's formula [4]. Also, it is to be mentioned that the proof presented below and the derived value of the infinite primorial differs slightly from that of Munoz and Marco [5]. This difference may be on account of the fact that we use the two related but slightly different formulas relating $\zeta(s)$ and $\zeta^{\prime}(s)$ - one given by lemma 2.1 and the other given in terms the Von Mangoldt function. Nonetheless, the result of 
the aforesaid paper and that obtained here point in the same direction. Now, the Von Mangoldt function is defined as

$$
\Lambda(n)= \begin{cases}\ln p, & \text { when } n=p^{k} \text { for some prime } p \text { and integer } k \geq 1 \\ 0, & \text { otherwise. }\end{cases}
$$

As mentioned earlier, we know that the Von Mangoldt function is related to the zeta function and its derivative as

$$
\frac{\zeta^{\prime}(s)}{\zeta(s)}=-\sum_{n=1}^{\infty} \frac{\Lambda(n)}{n^{s}}
$$

Thus, at $s=0$ one has

$$
\frac{\zeta^{\prime}(0)}{\zeta(0)}=-\sum_{n=1}^{\infty} \Lambda(n)
$$

Again, $\zeta(0)=-\frac{1}{2}$, and so we have

$$
2 \zeta^{\prime}(0)=\sum_{n=1}^{\infty} \Lambda(n)
$$

Now, the derivative of the zeta function is given as

$$
\zeta^{\prime}(s)=-\sum_{n=2}^{\infty} \frac{\ln n}{n^{s}}
$$

which yields at $s=0$

$$
\begin{gathered}
\zeta^{\prime}(0)=-\sum_{n=2}^{\infty} \ln n \\
\zeta^{\prime}(0)=-\ln \left[\prod_{n=2}^{\infty} n\right]
\end{gathered}
$$

which essentially implies the infinite factorial $(N !)$. Thus, the Van Mangoldt function would be given as

$$
\sum_{n=1}^{\infty} \Lambda(n)=-2 \ln N !
$$

Now, we know that $[6]$

$$
\frac{\zeta^{\prime}(0)}{\zeta(0)}=\ln 2 \pi
$$

Thus, we derive

$$
N !=\sqrt{2 \pi}
$$

which we mentioned earlier as deducible from Lerch's formula. This equation implies that the product of all natural numbers converges and attains the value of $\sqrt{2 \pi}$, on account of analytic continuation. Therefore, we have from Lemma 2.1 [at, $s=0$ ]

$$
-\frac{\zeta^{\prime}(0)}{\zeta(0)}=\sum_{n=1}^{\infty} \Lambda(n)=-\sum_{p} \ln p-\frac{\delta}{\zeta(0)}=-\sum_{p} \ln p+2 \delta
$$


which gives

$$
\ln N !=\frac{1}{2} \sum_{p} \ln p-\delta
$$

Thus, we have

$$
N !=\frac{1}{f(p)} \prod_{p} \sqrt{p}
$$

Again, from the relation $f(p)=\prod_{p} p^{l^{s} / r^{s}}$ we have $\left.f(p)\right|_{s=0}=\prod_{p} p^{1+1+\cdots}$. And, since the exponent $1+1+\cdots$ is the infinite sum

$$
1+1+\cdots=\zeta(0)=-\frac{1}{2}
$$

we finally obtain the desired result

$$
p \#=N !=\sqrt{2 \pi}
$$

Remark. Interestingly, since primes are fundamental natural numbers making up all other natural numbers, looking at the last equation one may say that the square of the infinite product of all primes represents the circumference of a Euclidean unit circle.

Remark. Incidentally, the infinite primorial has a resemblance with de Polignac or Legendre's formula

$$
k !=\prod_{p} p^{f(p, k)}
$$

with $k !=N$ ! and $f(p, k)=1$.

Corollary 2.1. The sum of the reciprocals of all the prime numbers diverges.

Proof. This is already a known result, but we merely prove it by resorting to Theorem $\mathbf{1}$. Let us consider the sum of the reciprocals of all primes:

$$
\sum_{n=1}^{\infty} \frac{1}{p_{n}}=\frac{1}{2}+\frac{1}{3}+\frac{1}{5}+\cdots
$$

This can also be written as

$$
\sum_{n=1}^{\infty} \frac{1}{p_{n}}=\frac{3.5 \cdot 7 \cdot \cdots+2 \cdot 5 \cdot 7 \cdot \cdots+2.3 .7 \cdot \cdots+\cdots}{2 \cdot 3 \cdot 5 \cdot 7 \cdot \cdots}
$$

Or,

$$
\sum_{n=1}^{\infty} \frac{1}{p_{n}}=\frac{\sum_{n=1}^{\infty}\left\{\prod_{p_{n} \backslash p_{i}} p_{n}\right\}}{\prod_{p} p}
$$

where, $\prod_{p_{n} \backslash p_{i}}$ implies that the product is taken over all primes up to the $n$-th prime except the $i-t h$ one. Now, using our result $\prod_{p} p=\sqrt{2 \pi}$ we have

$$
\sum_{n=1}^{\infty} \frac{1}{p_{n}}=\frac{1}{\sqrt{2 \pi}}\left[\sum_{n=1}^{\infty}\left\{\prod_{p_{n} \backslash p_{i}} p_{n}\right\}\right]
$$


Since, one distinct prime is excluded from each product term, it is evident that the sequence of the partial sums of the product terms do not approach a finite limit. Thus, it follows that

$$
\sum_{n=1}^{\infty} \frac{1}{p_{n}}=\infty
$$

i.e., the sum of the reciprocal of the prime numbers diverges. This is not a rigorous proof like that of Mertens [7], Furstenberg [8] or Erdos [9], but rather a simple application of Theorem 2.

Corollary 2.2. If $n$ represents all natural numbers, and then, if the infinite sum of the Von Mangoldt function over $n$ is expressible as

$$
\sum_{n=1}^{\infty} \Lambda(n)=\sum_{p} \ln p+g(p)=0.06
$$

where, $g(p)=\sum_{p} \ln p^{l_{i}}, l_{i}$ denoting the extra multiplicity of the $i-$ th prime after it has been included once in the first sum and both the sums on the right hand side are taken over all distinct primes, then

$$
\sum_{n=1}^{\infty} \Lambda(n)=\sum_{p} \ln p^{1 /(2 \pi)^{3 / 2}}
$$

Proof. Now, from equation (6) we have [at, $s=0]$

$$
\begin{gathered}
2 \sum_{n=1}^{\infty} \Lambda(n)=g(p)+2 \delta \\
-2 \ln N !=\frac{1}{2} g(p)+\ln f(p)
\end{gathered}
$$

which gives

$$
N !=\frac{1}{\sqrt{f(p)}} e^{-g(p) / 4}
$$

From this, using $N !=\sqrt{2 \pi}$ and $\left.f(p)\right|_{s=0}=\prod_{p} \frac{1}{\sqrt{p}}=(2 \pi)^{-1 / 4}$, one obtains the following result

$$
g(p)=\sum_{p} \ln p^{l_{i}}=-\frac{3}{2} \ln (2 \pi)
$$

which yields

$$
\sum_{n=1}^{\infty} \Lambda(n)=\sum_{p} \ln p^{1 /(2 \pi)^{3 / 2}} \approx 0.06
$$

Remark. The negative value of the function $g(p)$ is not surprising for infinite sums, since we know the following result by virtue of analytic continuation [6]: $\zeta(0)=1+1+\cdots=-\frac{1}{2}$. 


\section{The Chebyshev function and the totient function}

The first Chebyshev function $[10,11,12]$ and the Euler totient function are special functions that have several interesting implications in the field of number theory. The following results, including a lemma pertinent to Carmichael's totient function conjecture at the end of the current section, might further extend the existing applications.

Lemma 3.1. Consider some real number $x$. Given the first Chebyshev function

$$
v(x)=\sum_{p<x} \ln p
$$

it can be alternatively expressed as

$$
v(x)=\sum_{d \mid x} \Lambda(d)+\sum_{e \mid r} \Lambda(e)+\ln \left[\frac{\phi(l)}{\phi(x)}\right]
$$

where, $\prod_{p \nmid x} p=r, \phi(x)$ is the totient function and $\prod_{p \mid x}(p-1)=\prod_{p \mid x} \phi(p)=\phi(l)$.

Proof. The Chebyshev function can also be expressed as

$$
\prod_{p<x} p=e^{v(x)}
$$

Now, the left hand side of this equation can be expressed as below

$$
\prod_{p<x} p=\prod_{p \mid x} p \prod_{p \nmid x} p
$$

Again, we know the Euler totient function is given as

$$
\phi(x)=x \prod_{p \mid x}\left(1-\frac{1}{p}\right)
$$

which can also be written as

$$
\phi(x)=\frac{x \prod_{p \mid x}(p-1)}{\prod_{p \mid x} p}
$$

Thus, we have

$$
e^{v(x)}=\frac{x \prod_{p \mid x}(p-1)}{\phi(x)} \times \prod_{p \nmid x} p
$$

Now, it is known that

$$
\phi(p)=p-1
$$

And so, we have

$$
e^{v(x)}=\frac{x \prod_{p \mid x} \phi(p)}{\phi(x)} \times \prod_{p \nmid x} p
$$

From this we will have

$$
v(x)=\ln x+\ln \prod_{p \mid x} \phi(p)+\ln \prod_{p \nmid x} p-\ln \phi(x)
$$


Now, we know that for all divisors $d$ that divide $x$, one has the Von Mangoldt property

$$
\sum_{d \mid x} \Lambda(d)=\ln x
$$

Suppose the product $\prod_{p \nmid x} p=r$, then we also have

$$
\ln \prod_{p \nmid x} p=\ln r=\sum_{e \mid r} \Lambda(e)
$$

which yields the result

$$
v(x)=\sum_{d \mid x} \Lambda(d)+\sum_{e \mid r} \Lambda(e)+\ln \left[\frac{\prod_{p \mid x} \phi(p)}{\phi(x)}\right]
$$

Now, it is evident that for all primes, $p>2, \prod_{p \mid x} \phi(p)=\prod_{p \mid x}(p-1)$ will be an even number. Let this number be $\phi(l)$, so that we have

$$
v(x)=\sum_{d \mid x} \Lambda(d)+\sum_{e \mid r} \Lambda(e)+\ln \left[\frac{\phi(l)}{\phi(x)}\right]
$$

Theorem 2. The totient function of some real number $x$ can be expressed as

$$
\phi(x)=\phi(l) e^{\sum_{d \mid x} \Lambda(d)+\sum_{e \mid r} \Lambda(e)-\sum_{p<x} \Lambda\left(x_{p}\right)}
$$

Also

$$
\phi(x)=x \phi(l) e^{-\sum_{p \mid x} \Lambda(p)}
$$

Proof. From the definition of the Von Mangoldt function we know that for some distinct prime $p$, we shall have

$$
e^{\Lambda\left(x_{p}\right)}=p
$$

where, $x_{p}=p^{k}\left(x_{p}>x\right.$ or, $\left.x_{p} \leq x\right)$. Thus, we have

$$
\prod_{p_{i} \leq x} p_{i}=e^{\sum_{p_{i} \leq x} \Lambda\left(x_{p}\right)}
$$

This is essentially the expression for the Chebyshev function

$$
v(x)=\ln \prod_{p_{i} \leq x} p_{i}
$$

Thus

$$
v(x)=\sum_{p \leq x} \Lambda\left(x_{p}\right)
$$


where, the $p$ 's are distinct. Thus, we have from Lemma $\mathbf{3 . 1}$

$$
\sum_{p<x} \Lambda\left(x_{p}\right)-\sum_{d \mid x} \Lambda(d)-\sum_{e \mid r} \Lambda(e)=\ln \left[\frac{\phi(l)}{\phi(x)}\right]
$$

from which we derive the following relation

$$
\phi(x)=\phi(l) e^{\sum_{d \mid x} \Lambda(d)+\sum_{e \mid r} \Lambda(e)-\sum_{p<x} \Lambda\left(x_{p}\right)}
$$

Again, we had previously obtained the relation

$$
\phi(x)=\frac{x \prod_{p \mid x} \phi(p)}{\prod_{p \mid x} p}
$$

Suppose, there are primes ' $p$ ' conforming with the Von Mangoldt function property

$$
\ln x=\sum_{p \mid x} \Lambda(p)
$$

But, by definition of the Von Mangoldt function: $\Lambda(p)=\ln p$, where $p=p^{k}$ and $k=1$. So

$$
\sum_{p \mid x} \Lambda(p)=\ln \prod_{p \mid x} p
$$

Again, writing $\prod_{p \mid x} \phi(p)=\phi(l)$, we shall finally derive from equation (15)

$$
\phi(x)=x \phi(l) e^{-\sum_{p \mid x} \Lambda(p)}
$$

Lemma $\mathbf{3 . 2}$ (On Carmichael's conjecture). Let $x$ and $y$ be integers such that $x \neq y$. If $\phi(x)=$ $\phi(y)$ and if one of the integers is prime, then the other cannot be a prime.

Proof. This easily follows from Theorem 2. For two different integers $x$ and $y$ we have

$$
\begin{aligned}
& \phi(x)=x \prod_{p \mid x} \phi(p) e^{-\sum_{p \mid x} \Lambda(p)} \\
& \phi(y)=y \prod_{p \mid y} \phi(p) e^{-\sum_{p \mid y} \Lambda(p)}
\end{aligned}
$$

Suppose, both $x$ and $y$ are primes with $x=p_{1}$ and $y=p_{2}$, then we have

$$
p_{1} \phi\left(p_{1}\right) e^{-\ln \left(p_{1}\right)}=p_{2} \phi\left(p_{2}\right) e^{-\ln \left(p_{2}\right)}
$$

which yields the result

$$
\phi\left(p_{1}\right)=\phi\left(p_{2}\right)
$$

Since, equation (17) is not possible, we have the lemma substantiated. Also, it insinuates from this that: if there are integers such that $x_{1} \neq x_{2} \cdots \neq x_{n}$ with $\phi\left(x_{2}\right)=\phi\left(x_{2}\right) \cdots=\phi\left(x_{n}\right)$ and if one of them is a prime then the others cannot be primes. 


\section{The prime counting function}

This section concerns with the prime counting function that has been investigated by several authors $[13,14,15,16,17,18,19,20,21,22,23]$ and some identities and approximations that provide novel expressions for the aforementioned special function. These expressions might help researchers to find more precise values for $\pi(x)$, confirm known and determine hitherto unknown properties of prime numbers.

Lemma 4.1. Given the totient function

$$
\phi(L)=\prod_{p \leq x}(p-1)=\prod_{p \leq x} \phi(p)
$$

where, $p$ denotes primes, the prime counting function can be approximated as

$$
\pi(x) \approx x(x-1)\left[v^{\prime}(x)-\frac{\phi^{\prime}(L)}{\phi(L)}\right]
$$

for large values of $x$, where, the primes (I) denote derivatives with respect to $x$.

Proof. Now, we know that the zeta function is related to the Euler product as

$$
\zeta(s)=\prod_{p} \frac{1}{1-p^{-s}}
$$

So, at the point $s=1$, we would have the relation

$$
\prod_{p} p=\zeta(1) \prod_{p}(p-1)
$$

Again, the zeta function is related to the prime-counting function $(\pi(x))$ as

$$
\ln \zeta(s)=s \int_{0}^{\infty} \frac{\pi(x)}{x\left(x^{s}-1\right)} \mathrm{d} x
$$

where, $x$ represents real numbers. Thus, at $s=1$ this relation becomes

$$
\ln \zeta(1)=\int_{0}^{\infty} \frac{\pi(x)}{x(x-1)} \mathrm{d} x
$$

Thus, we have

$$
\ln \left[\frac{\prod_{p} p}{\prod_{p}(p-1)}\right]=\int_{0}^{\infty} \frac{\pi(x)}{x(x-1)} \mathrm{d} x
$$

This can also be written as

$$
\ln \left[\frac{\prod_{p \leq x} p \prod_{p>x} p}{\prod_{p \leq x}(p-1) \prod_{p>x}(p-1)}\right]=\int_{0}^{\infty} \frac{\pi(x)}{x(x-1)} \mathrm{d} x
$$

Now, for large values of $x$ the primes $p>x$ will be very large. So

$$
\frac{\prod_{p>x} p}{\prod_{p>x}(p-1)}=\frac{1}{\prod_{p>x}(1-1 / p)} \approx 1
$$

Thus, we derive

$$
\int_{0}^{\infty} \frac{\pi(x)}{x(x-1)} \mathrm{d} x \approx \ln \prod_{p \leq x} p-\ln \prod_{p \leq x}(p-1)
$$


where, the first term on the right hand side is the first Chebyshev function and the second term is the logarithm of the totient function of a prime number, both being monotonically increasing functions. So, without loss of generality, we can write:

$$
\int_{0}^{\infty} \mathrm{d}[v(x)-\ln \phi(L)]=\ln \prod_{p \leq x} p-\ln \prod_{p \leq x}(p-1)
$$

Thus, we obtain the following expression for the prime counting function

$$
\pi(x) \approx x(x-1)\left[v^{\prime}(x)-\frac{\phi^{\prime}(L)}{\phi(L)}\right]
$$

Remark. It is to be borne in the mind that, $\phi(L)$ and $\phi(l)$ may or may not be the same, for obvious reasons.

Theorem 3. For considerably large real values of $x$, the ratio of the prime counting function and the totient function can be expressed as

$$
\frac{\pi(x)}{\phi(x)} \approx \prod_{p \leq x, p \nmid x}\left(1-\frac{1}{p}\right)
$$

where, the product is taken over primes less than or equal to $x$, that do not divide $x$.

Proof. Now, equation (18) can also be written as

$$
\pi(x)=x(x-1) \frac{\mathrm{d}}{\mathrm{d} x}\left[\ln \left\{\frac{\prod_{p \leq x} p}{\prod_{p \leq x}(p-1)}\right\}\right]
$$

and, the prime number theorem gives the asymptotic relation

$$
\pi(x) \sim \frac{x}{\ln x}
$$

Using this relation in the previous equation, we have

$$
\frac{1}{(x-1) \ln x} \sim \frac{\mathrm{d}}{\mathrm{d} x}\left[\ln \left\{\frac{\prod_{p \leq x} p}{\prod_{p \leq x}(p-1)}\right\}\right]
$$

Since, $x$ is considerably large, we take $x-1 \approx x$. Thus, we have

$$
\frac{1}{x \ln x} \sim \frac{\mathrm{d}}{\mathrm{d} x}\left[\ln \left\{\frac{\prod_{p \leq x} p}{\prod_{p \leq x}(p-1)}\right\}\right]
$$

Writing, $\frac{\prod_{p \leq x} p}{\prod_{p \leq x}(p-1)}=a(x)$, we find that

$$
a(x)=\frac{\prod_{p \leq x} p}{\prod_{p \leq x}(p-1)} \approx \ln x
$$

since, $\frac{\mathrm{d}}{\mathrm{d} x}[\ln (\ln x)]=\frac{1}{x \ln x}$. Thus

$$
\frac{1}{\prod_{p \leq x, p \mid x}(1-1 / p) \prod_{p \leq x, p \nmid x}(1-1 / p)} \approx \ln x
$$


Now, using the property of the totient function given in (10) we have

$$
\frac{x}{\phi(x) \prod_{p \leq x, p \nmid x}(1-1 / p)} \sim \ln x
$$

which finally yields the result, after using the prime number theorem

$$
\frac{\pi(x)}{\phi(x)} \sim \prod_{p \leq x, p \nmid x}\left(1-\frac{1}{p}\right)
$$

valid for considerably large values of $x$.

Theorem 4. Consider the following function

$$
\tau(x)=\int_{1}^{x} \frac{\mathrm{d} t}{\ln t}
$$

If we choose the variable $t=p^{k}$, then the logarithmic integral function

$$
l i(x)=\tau(x)
$$

and also, li $(x)$ is zero at $x=1$, i.e., $\operatorname{li}(1)=\int_{0}^{1} \frac{\mathrm{d} t}{\ln t}=0$.

Considering the second Chebyshev function

$$
\psi(x)=\sum_{p^{k} \leq x} \ln p
$$

the prime counting function will be given as (with $p$ denoting primes)

$$
\pi(x) \approx \begin{cases}\frac{1}{k}\left[l i(X)-l i(2)^{\frac{1}{k}}\right]+\frac{X}{k}+O\left(\sqrt{X^{k}}\right), & \text { when } x \ln x \gg x\left(\text { with } X=x^{\frac{1}{k}}\right) ; \\ L i(x)-\frac{2}{\ln 2}+O(\sqrt{x}), & \text { for, } x \gg a x^{\frac{1}{a}} ; \\ a(a-1) x^{a}-\ln a \ln \ln x-\ln a\left[\frac{\ln ^{2} \ln x-\ln ^{2} \ln 2}{2}\right] & \\ +a(a-1)\left[l i\left(x^{a}\right)-\operatorname{li}\left(2^{a}\right)\right]+O(\sqrt{x}), & \text { for, } \ln x \gg \frac{1}{a} \text { and } x \gg \frac{\ln a}{a}+1 .\end{cases}
$$

where, $k \geq 1$ and $a \geq 2$ are finite positive integers, with Li(x) being the offset logarithmic integral function and $O$ being the big $O$-notation.

Also, considering the first Chebyshev function

$$
v(x)=\sum_{p \leq x} \ln p
$$

the prime counting function for large values of $x$ is given as

$$
\pi(x) \approx x\left[\ln \ln x-x \frac{\phi^{\prime}(L)}{\phi(L)}\right]
$$

Proof. Now, we have for the variable $t$

$$
t=p^{k} \Rightarrow \ln t=\ln p^{k}
$$

Thus

$$
\mathrm{d} t=p^{k} \ln p \mathrm{~d} k
$$


Using this substitution we have for the function $\tau(x)$

$$
\tau(x)=\int_{0}^{\ln x / \ln p} \frac{p^{k}}{k} \mathrm{~d} k
$$

which gives

$$
\tau(x)=E i\left[\ln p^{k}\right]_{0}^{\ln x / \ln p}
$$

This finally yields

$$
\tau(x)=E i[\ln x]=l i(x)
$$

$E i(x)$ being the exponential integral function. This implies that

$$
l i(1)=\int_{0}^{1} \frac{\mathrm{d} t}{\ln t}=0
$$

Now, we have considered the substitution

$$
\ln t=\ln p^{k}
$$

Again, if $p$ denotes a prime, we have a similar equation as above that is related to the second Chebyshev function defined in the following manner:

$$
\psi\left(x_{1}\right)=\ln t_{1}=\ln p_{1}
$$

where, $p_{1}^{k} \leq x_{1}$. Summing over all such primes, one has

$$
\sum_{t} \ln t=\sum_{p^{k} \leq x} \ln p
$$

and considering

$$
\psi(x)=\sum_{i} \psi\left(x_{i}\right)
$$

we can consider either of the following three cases

$$
\begin{gathered}
\psi(x)=\sum_{t} \ln t \approx \int_{x^{1 / k}}^{x^{1-\epsilon}} \ln p \mathrm{~d} p \\
\psi(x)=\sum_{t} \ln t \approx \int_{1}^{a}\left[\int_{x^{1-\epsilon}}^{x^{1 / k}} \ln p \mathrm{~d} p\right] \mathrm{d} k
\end{gathered}
$$

or,

$$
\psi(x)=\sum_{t} \ln t \approx \int_{1}^{a}\left[\int_{x^{1-\epsilon}}^{\ln x^{1 / k}} \ln p \mathrm{~d} p\right] \mathrm{d} k
$$

where, $\epsilon$ is arbitrary, $a$ is some finite positive number and $k$ is some finite positive integer (since an infinite value of $k$ would annul the feasibility of the inequality $p^{k} \leq x$ ). So, considering equation (21) where $k$ can be arbitrarily chosen, we have

$$
\psi(x) \approx x^{1-\epsilon}\left[\ln (x)^{1-\epsilon}-1\right]-\frac{x^{1 / k}}{k}[\ln x-k]
$$


Therefore, we have

$$
\frac{\psi(x)}{x} \approx\left[\frac{\ln (x)^{1-\epsilon}}{x^{\epsilon}}-\frac{1}{x^{\epsilon}}\right]-\frac{1}{k}\left[\frac{\ln x}{x^{1-\frac{1}{k}}}-\frac{1}{x^{1-\frac{1}{k}}}\right]
$$

Thus, considering $\epsilon$ and $k$ to be finite, it is easy to see that all the terms vanish for $x \rightarrow \infty$ which is essentially a dilemma. However, since $\epsilon$ is arbitrary this dilemma can be remedied by firstly considering $0<\epsilon<1$ and sufficiently small such that

$$
\left.x^{-\left(x^{\epsilon}+\epsilon\right.}\right) \approx e
$$

i.e., the left hand side approaches the Euler number when $x \rightarrow \infty$. Secondly, we take $k \approx 1$. Thus, we will have from equation (25)

$$
\begin{aligned}
\frac{\psi(x)}{x} \approx & {\left[\frac{\ln x\left(1-x^{\epsilon}-\epsilon\right)-1}{x^{\epsilon}}\right]+\left[\frac{1}{k} \frac{1}{x^{1-\frac{1}{k}}}\right]_{k \approx 1} } \\
& \Rightarrow \frac{\psi(x)}{x} \approx\left[\ln \left\{x^{-\left(x^{\epsilon}+\epsilon\right)}\right\}-1\right]+1
\end{aligned}
$$

with $x^{\epsilon}+\epsilon \gg 1$. Therefore, we finally have

$$
\lim _{x \rightarrow \infty} \frac{\psi(x)}{x} \approx 1
$$

which is the prime number theorem, itself. Even so, it must be noted that the sufficiently small value of $\epsilon$ was accommodated for the scenario when $x \rightarrow \infty$, in order that the prime number theorem is satisfied. It can still be arbitrarily changed. Now, we can see that in the following

$$
\psi(x) \approx \int_{x^{1 / k}}^{x^{1-\epsilon}} \ln p \mathrm{~d} p=x^{1-\epsilon}\left[\ln (x)^{1-\epsilon}-1\right]-\frac{x^{1 / k}}{k}[\ln x-k]
$$

the integration limits are taken backwards. Again, the prime counting function is related to the Chebyshev function as

$$
\Pi(x)=\int_{2}^{x} \frac{\psi(t) \mathrm{d} t}{t \ln ^{2} t}+\frac{\psi(x)}{\ln x}
$$

where, $\Pi(x)=\sum_{n \leq x} \frac{\Lambda(n)}{\ln n}$. Here, a negative sign must be introduced on account of the backward integration limits mentioned before, to make the above equation feasible with respect to the expression of $\psi(x)$ in (24). Incidentally, considering equation (24) [with the arbitrary value of $\epsilon=1$ and taking $\left.\frac{x^{1 / k}}{k}[\ln x-k] \gg 1(>0)\right]$, we have

$$
\Pi(x) \approx \int_{2}^{x} \frac{t^{\frac{1}{k}-1} \mathrm{~d} t}{k \ln t}+\frac{x^{1 / k}}{k}
$$

which yields

$$
\Pi(x) \approx \frac{1}{k}\left[E i\left(\ln t^{\frac{1}{k}}\right)\right]_{2}^{x}+\frac{x^{1 / k}}{k}
$$

And from this, we finally derive

$$
\Pi\left(X^{k}\right) \approx \frac{1}{k}\left[\operatorname{li}(X)-\operatorname{li}\left(2^{\frac{1}{k}}\right)\right]+\frac{X}{k}
$$


where, we have written: $x^{\frac{1}{k}}=X$. Again, we know the following relation

$$
\pi\left(X^{k}\right)=\Pi\left(X^{k}\right)+O\left(\sqrt{X^{k}}\right)
$$

Thus, we obtain the following result valid for considerably large values of $x\left(=X^{k}\right)$

$$
\pi(x) \approx \frac{1}{k}\left[\operatorname{li}(X)-\operatorname{li}\left(2^{\frac{1}{k}}\right)\right]+\frac{X}{k}+O\left(\sqrt{X^{k}}\right)
$$

Incidentally, here we can once again consider the case: $k \approx 1$, without loss of any generality. Therefore, remembering $\operatorname{Li}(x)=l i(x)-l i(2)$ we would have

$$
\pi(x) \approx \operatorname{Li}(x)+x+O(\sqrt{x})
$$

And this equation shall be the pedestal for the first route to the Riemann hypothesis, in the subsequent section.

Now, we shall consider equation (22) where $k$ is a variable and hence the double integration. Carrying out the integration we would have

$$
\begin{gathered}
\psi(x) \approx-\ln x\left[\operatorname{Ei}\left(\ln x^{\frac{1}{k}}\right)\right]_{1}^{a}-\left[k x^{\frac{1}{k}}\right]_{1}^{a}+\left[\operatorname{Ei}\left(\ln x^{\frac{1}{k}}\right)\right]_{1}^{a}-x^{1-\epsilon}\left[\ln (x)^{1-\epsilon}-1\right](a-1) \\
\psi(x) \approx x-a x^{\frac{1}{a}}-x^{1-\epsilon}\left[\ln (x)^{1-\epsilon}-1\right](a-1)
\end{gathered}
$$

Thus, we have

$$
\frac{\psi(x)}{x} \approx 1-\frac{a}{x^{1-\frac{1}{a}}}-\left[\frac{\ln (x)^{1-\epsilon}}{x^{\epsilon}}-\frac{1}{x^{\epsilon}}\right](a-1)
$$

Considering $a$ to be finite and $\epsilon \rightarrow \infty$, we have

$$
\lim _{x \rightarrow \infty} \frac{\psi(x)}{x} \approx 1
$$

Thus, we obtain

$$
\lim _{x \rightarrow \infty} \frac{\psi(x)}{x} \approx 1
$$

which is again the prime number theorem. And, since $a$ is some finite positive integer and $\epsilon \rightarrow \infty$, equation (30) becomes

$$
\psi(x) \approx x-a x^{\frac{1}{a}}
$$

Now, as before, considering the last equation and $x \gg a x^{\frac{1}{a}}$ we have

$$
\Pi(x) \approx \int_{2}^{x} \frac{\mathrm{d} t}{\ln ^{2} t}+\frac{x}{\ln x}
$$

or,

$$
\Pi(x) \approx \operatorname{Li}(x)-\frac{2}{\ln 2}
$$

And, thus we finally have the expression

$$
\pi(x) \approx \operatorname{Li}(x)-\frac{2}{\ln 2}+O(\sqrt{x})
$$


Now, let us consider equation (23). We have

or,

$$
\begin{aligned}
\psi(x) \approx & -\ln x\left[\frac{\ln ^{2} k}{k}\right]_{1}^{a}+\ln x \ln \ln x[\ln k]_{1}^{a} \\
& -\ln x[\ln k]_{1}^{a}-x^{1-\epsilon}\left[\ln (x)^{1-\epsilon}-1\right](a-1)
\end{aligned}
$$

$$
\psi(x) \approx \ln x \ln a\left[\ln \ln x-\frac{\ln a}{a}-1\right]-x^{1-\epsilon}\left[\ln (x)^{1-\epsilon}-1\right](a-1)
$$

Here, the first term on the right hand side is easy to understand when we evaluate $\lim _{x \rightarrow \infty} \frac{\psi(x)}{x}$. So, considering $\epsilon=1-a$ and $a$ to be sufficiently small and close to 1 (but $>1$ ) such that

$$
(x)^{-x^{a-1}(a-1)\left(a-\frac{1}{\ln x}\right)} \approx e
$$

(i.e., the left hand side approaching the Euler number when $x \rightarrow \infty$ ) we have for the second term on the right hand side

$$
x^{1-\epsilon}\left[\ln (x)^{1-\epsilon}-1\right](a-1)=x\left[\frac{\left(a-\frac{1}{\ln x}\right)(a-1)}{\frac{1}{x^{a-1} \ln x}}\right]=-x \ln \left[(x)^{-x^{a-1}(a-1)\left(a-\frac{1}{\ln x}\right)}\right]
$$

Again, by our considerations we shall have

$$
\ln \left[(x)^{-x^{a-1}(a-1)\left(a-\frac{1}{\ln x}\right)}\right] \approx 1
$$

And consequently, we can write the following

$$
\lim _{x \rightarrow \infty} \frac{\psi(x)}{x} \approx 1
$$

as expected. It is obvious that like equation (21) we would have a backward integration. Thus, using $\epsilon=1-a, \ln x^{a} \gg 1$ and $\ln \ln x \gg \frac{\ln a}{a}+1$ and considering a negative sign as in the preceding cases, we have

$$
\Pi(x) \approx a(a-1) x^{a}-\ln a \int_{2}^{x} \frac{\ln \ln t \mathrm{~d} t}{t \ln t}+a(a-1) \int_{2}^{x} \frac{t^{a-1}}{\ln t}-\ln a \ln \ln x
$$

It is easy to see that the above equation simplifies as

$$
\begin{aligned}
\Pi(x) \approx & a(a-1) x^{a}-\ln a \ln \ln x-\ln a\left[\frac{\ln ^{2} \ln x-\ln ^{2} \ln 2}{2}\right] \\
& +a(a-1)\left[l i\left(x^{a}\right)-\operatorname{li}\left(2^{a}\right)\right]
\end{aligned}
$$

and therefore, the prime counting function is given as

$$
\begin{aligned}
\pi(x) \approx & a(a-1) x^{a}-\ln a \ln \ln x-\ln a\left[\frac{\ln ^{2} \ln x-\ln ^{2} \ln 2}{2}\right] \\
& +a(a-1)\left[l i\left(x^{a}\right)-\operatorname{li}\left(2^{a}\right)\right]+O(\sqrt{x})
\end{aligned}
$$

Now, let us consider the first Chebyshev function just like the second, in the following manner

$$
v\left(x_{1}\right)=\ln t_{1}=\ln p_{1}
$$


Summing over all primes $p \leq x$, we have

$$
v(x)=\sum_{i} v\left(x_{i}\right)=\sum_{t} \ln t=\sum_{p \leq x} \ln p
$$

As before, taking integration instead of the sum, we obtain

$$
v(x)=\sum_{t} \ln t \approx \int_{1}^{a}\left[\int_{\ln x}^{x^{1-\epsilon}} \ln p \mathrm{~d} p\right] \mathrm{d} k
$$

From this we get the following expression for $v(x)$

$$
v(x) \approx x^{1-\epsilon}\left[\ln x^{1-\epsilon}-1\right]-\ln x[\ln \ln x-1]
$$

It is easy to see that we obtain the following relation

$$
\lim _{x \rightarrow \infty} \frac{v(x)}{x} \approx \frac{1}{\epsilon}
$$

Choosing $\epsilon=1$ (since it is arbitrary), we finally have

$$
\lim _{x \rightarrow \infty} \frac{v(x)}{x} \approx 1
$$

which is another form of the prime number theorem, with regards to the first Chebyshev function. As usual, on account of backward integration limits, considering a negative value (for feasibility) and Lemma $\mathbf{4 . 1}$ we have the following relation between $\pi(x)$ and $v(x)$ (with $\epsilon=1$ and for large values of $x$ )

$$
\pi(x) \approx x\left[\ln \ln x-x \frac{\phi^{\prime}(L)}{\phi(L)}\right]
$$

From the above deductions and results we are in a position to conclude that the second Chebyshev can have multiple representations that conform with the prime number theorem. Equations (21), (22) and (23) are such representations. Incidentally, these representations yield different expressions for $\pi(x)$ through equations (28), (33) and (38).

We have also found a representation for the first Chebyshev function that too conforms with the prime number theorem and the expression has brought another expression (42) for the prime counting function in terms of the Euler totient function. Essentially, we have found representations that define the prime counting function in different ways, subject to some conditions.

\section{Discussions}

The expression relating $\zeta^{\prime}(s)$ to $\zeta(s)$ might have interesting implications in future research endeavours apposite to the zeta function, and, the same can be said for the expression of $\sum_{n=0}^{\infty} \frac{\zeta(n s)}{n !}$.

It is worth mentioning and quite ostensible that the most surprising result of the current article is Theorem 2, that substantiates the convergence of the infinite primorial that incidentally coincides with the infinite factorial. Further research and investigations are necessary to see the implications of such a counterintuitive result, though the result points toward the same direction as Munoz and Marco [5].

The results of Lemma $\mathbf{3 . 1}$ and Theorem 3 yield novel expressions through which the first Chebyshev function, the Von Mangoldt function and the totient function are related. Essentially, 
these relations might prove to be prospective advancements for future research.

Lemma 4.1. Theorem 3 and Theorem 4 concern with the prime counting function and hence they might provide significant insights in determining $\pi(x)$ or studies related to other special functions like the zeta function.

\section{References}

[1] G. F. B Riemann, Uber die Anzahl der Primzahlen unter einer gegebenen Grosse, Monatsber. Konigl. Preuss. Akad. Wiss., Berlin, 671-680, Nov, 1859.

[2] J. Borwein et. al., Computational Strategies for the Riemann Zeta Function, J. Comp. App. Math., 121 (1-2): 247-296, 2000.

[3] H. von Mangoldt, Zu Riemanns Abhandlungen "Ueber die Anzahl der Primzahlen unter einer gegebenen Grosse", J. Reine Angew. Math., 114 (1895), pp. 255-305.

[4] N. Kurokawa \& M. Wakayama, A generalization of Lerch's formula, Czechoslovak Math. J. 54, 941-947, 2004.

[5] E. Muñoz García \& R. Pérez Marco, The Product Over All Primes is $4 \pi^{2}$, Communications in Mathematical Physics, vol. 277, pages 69-81 (2008).

[6] J. Sondow, Analytic continuation of Riemann's zeta function and values at negative integers via Euler's transformation of series, Proc. Amer. Math. Soc., 120: 421-424, 1994.

[7] F. Mertens, Ein Beitrag zur analytischer Zahlentheorie, J. Reine Angew. Math., 78: 46-62, 1874 .

[8] H. Furstenberg, On the infinitude of primes, American Mathematical Monthly, 62: 353, 1955 .

[9] P. Erdos, Uber die Reihe sum 1/p . (On the series sum 1/p .), Mathematica (Zutphen) B 7 , 1-2 (1938).

[10] G. H.Hardy and E. M. Wright, 'The Functions $\theta(x)$ and $\psi(x)$ ' and 'Proof that $\theta(x)$ and $\psi(x)$ are of Order $x$ ', An Introduction to the Theory of Numbers, $5^{\text {th }}$ ed. Oxford, England: Clarendon Press, pp. 340-342, 1979 .

[11] J. B. Rosser and L. Schoenfeld, Sharper Bounds for Chebyshev Functions $\theta(x)$ and $\psi(x)$, Math. Comput., 29, 243-269, 1975.

[12] L. Schoenfeld, Sharper Bounds for Chebyshev Functions $\theta(x)$ and $\psi(x)$, II, Math. Comput., 30, 337-360, 1976 .

[13] S. Ramanujan, Highly composite numbers, Proc. London Math. Soc., Series 2. 14: 347-409, 1915 .

[14] S. Ramanujan, Highly composite numbers, The Ramanujan Journal, 119-153, 1997.

[15] G. H. Hardy, A Formula of Ramanujan in the Theory of Primes, Journal of the London Mathematical Society, Volume s1-12, Issue 2, Pages 94-98, 1937.

[16] G. H. Hardy \& S. Ramanujan, On the normal number of prime factors of a number $\mathrm{n}$, Quart. J. Math., vol. 48, 76-92, 1917.

[17] B. C. Berndt, Ramanujan's Notebooks, Part I, Springer-Verlag, New York, 1985.

[18] A. de Regla, Formulas for $\pi(x)$ and the $n$th Prime, International Journal of Mathematics and Computer Science, 9(2014), 95-98. 
[19] M. Deleglise and J. Rivat, Computing $\pi(x)$ : The Meissel, Lehmer, Lagarias, Miller, Odlyzko Method, Math. Comput., 65, 235-245, 1996.

[20] J. Lagarias and A. Odlyzko, Computing $\pi(x)$ : An Analytic Method, J. Algorithms, 8, 173-191, 1987 .

[21] J. Buthe, An Improved Analytic Method for Calculating $\pi(x)$, Manuscripta Mathematica, volume 151, 329-352 (2016).

[22] D. J. Platt, Computing $\pi(x)$ analytically, Math. Comp., 84, 1521-1535, 2015.

[23] C. Axler, On a Sequence Involving Prime Numbers, Journal of Integer Sequences, Vol. 18 (2015), Article 15.7.6. 